\title{
La enseñanza de la Comunicación en Internet: niveles y orientación de la oferta formativa
}

\author{
María CADilla BAZ \\ Universidad Complutense de Madrid \\ mcadilla@ccinf.ucm.es
}

Recibido: $31 / 07 / 2012$

Aceptado: 26/10/2012

\section{Resumen}

Se analiza la oferta formativa en Comunicación disponible en la Red, teniendo en cuenta los niveles formativos y los promotores de los estudios, así como la orientación de los mismos y los ámbitos de aplicación profesional hacia los que se dirigen.

Palabras clave: Comunicación, Internet, Oferta educativa

\section{The education of the Communication in Internet: levels and orientation of the formative offer}

\begin{abstract}
This article analyzes the educational programs in Communication available on the Internet, taking into account the levels of education, the promoters of such studies, as well as their orientation and the fields of professional application to which they are addressed.

Keywords: Communication, Internet, Educational Offer

\section{Referencia normalizada}

CADILLA BAZ, María (2012): "La enseñanza de la Comunicación en Internet: niveles y orientación de la oferta formativa". Estudios sobre el mensaje periodístico. Vol. 18, núm. especial octubre, págs.: 191201. Madrid, Servicio de Publicaciones de la Universidad Complutense.
\end{abstract}

Sumario: 1. Introducción. 2. Metodología. 3. Desarrollo; 3.1. Niveles que ofrecen los estudios; 3.2. Oferta formativa en Comunicación según los promotores y ejecutores de los estudios; 3.3. Oferta formativa según la orientación de los estudios de Comunicación; 3.4. Oferta formativa según los ámbitos de aplicación de la actividad. 4. Conclusiones. 5. Referencias bibliográficas

\section{Introducción}

La progresiva implantación de las TIC se advierte de manera muy especial en el ámbito educativo, transformando completamente desde las metodologías de estudio hasta la propia oferta formativa en todos sus niveles.

Otra de las consecuencias de la conjunción entre Educación e Internet ha sido la incursión de las formas no regladas del conocimiento en la formación oficial. Las Universidades ya no son las únicas instituciones que ofrecen formación, y tampoco las únicas que hacen uso de los canales virtuales para su difusión, promoción y ejecución. El ciudadano tiene hoy a su alcance multitud de opciones para la adquisición de conocimientos, que ya no se limitan solamente a las fórmulas regladas u oficiales.

Hace tres décadas, Martín Serrano señalaba que la comunicación era "un tópico muy generalizado para referirse a casi todo" y que "el enfoque científico de la Teoría de la Comunicación está siendo utilizado fuera de su ámbito específico, tanto en el dominio de otras ciencias como en el de diversas prácticas cotidianas" (Martín et al., 1982: 68). En esta era digital en la que nos encontramos podemos decir sin lugar a 
dudas que estas afirmaciones están vigentes hoy más que nunca. Aunque los estudios comunicativos siguen siendo soporte fundamental de titulaciones específicas, también se han incorporado como materias en otros muchos estudios y tanto sus técnicas como sus aplicaciones forman parte en nuestros días de la mayoría de las actividades económicas, políticas, profesionales, culturales y, por supuesto, también de las educativas.

Este crecimiento a nivel cuantitativo está repercutiendo en una transformación sustancial de los estudios de la comunicación social, auspiciado por otro cambio cualitativo: los expertos en comunicación ya no son personal vinculado a las Administraciones públicas o al denominado Cuarto sector. Cada vez más, la empresa privada demanda comunicólogos, expertos en las tareas de la economía globalizada y competentes en las nuevas tecnologías.

\section{Metodología}

Este artículo trata de mostrar cuál es y cómo se construye la oferta formativa en Comunicación en nuestro país disponible a través de Internet, en un momento en que confluyen dos circunstancias de gran trascendencia: la incorporación masiva de las TIC al ámbito educativo y la adaptación de los estudios universitarios al Espacio Europeo de Educación Superior (EEES).

Los aspectos aquí tratados constituyen solamente una pequeña parte de los resultados de una amplia investigación, titulada La enseñanza reglada y no reglada de la comunicación en Internet y desarrollada por el grupo de Investigación "Identidades sociales y Comunicación" de la UCM (programa competitivo, ref. GR58/08).

La metodología empleada en dicha investigación consistió en el análisis de contenido de las páginas web de todos aquellos programas formativos ofrecidos en España, que impartían enseñanzas (regladas y no regladas) relacionadas con la Comunicación, con sitio web activo en el momento de la búsqueda y que aparecían como resultados del buscador Google.

Se trabajó con la totalidad del Universo encontrado (673 programas formativos), lo que ha permitido despojarse de los sesgos naturales implícitos en todo trabajo muestral. Por otra parte, aunque el uso de metodologías experimentales con herramientas web introduce otro sesgo inevitable, inherente a los criterios de selección y modos de operación de buscadores o motores de búsqueda (Pedro, 2012), considerando que Google acapara el 96,53\% de la cuota de mercado en España ${ }^{1}$, podemos considerar este sesgo inapreciable.

El modelo y los protocolos de análisis fueron diseñados ad hoc para la investigación. Consideramos que la radiografía que se ofrece del panorama formativo en Comunicación en Internet, puede resultar muy útil como punto de partida para monitorizar la evolución en el tiempo de las relaciones entre Internet y dichos estudios.

1 Datos de buscadores en España, enero 2012. Disponible en: www.josegalan.es/datos-debuscadores-espana-enero-2012 [Consultado el 13 de septiembre de 2012]. 


\section{Desarrollo}

\subsection{Niveles que ofrecen los estudios}

Presente en todas las actividades productivas y reproductivas de nuestra sociedad, la Red se ha convertido en aliado imprescindible y fundamental del ámbito educativo y una herramienta tan poderosa que está transformando desde las metodologías de estudio hasta los propios contenidos y estrategias docentes.

Sin embargo, el concepto de enseñanza virtual abriga múltiples y diversos modelos de enseñanza, por lo que cabe matizar que el uso de las tecnologías no siempre se traduce necesariamente en una enseñanza on line. Un modo de estar en Internet consiste en utilizar la Red como instrumento publicitario o de difusión de contenidos; el otro implica el uso de la Red como propia plataforma de trabajo y de negocios. Esta diferenciación permite distinguir dos tipos de acciones formativas no excluyentes: las que se desarrollan fuera de Internet pero que utilizan la Red como vehículo de promoción y divulgación y aquellas otras que ofrecen la formación directamente on line. Se incluyen aquí ambos tipos de propuestas, registrándose 232 programas formativos que hacen referencia al mundo on line y otros 350 que se desarrollan fuera de la Red pero que hacen uso de ella para darles difusión o como complemento a la formación presencial.

Más de la mitad de la oferta formativa en Comunicación que se ofrece a través de Internet se corresponde con titulaciones universitarias (201 titulaciones), si bien la Red aglutina también un considerable volumen de estudios no reglados: la tercera parte de los estudios localizados se desarrolla en forma de Cursos (212), Seminarios y Talleres (23), cerrando el repertorio de acciones formativas disponibles un pequeño grupo (20) de denominaciones diversas.

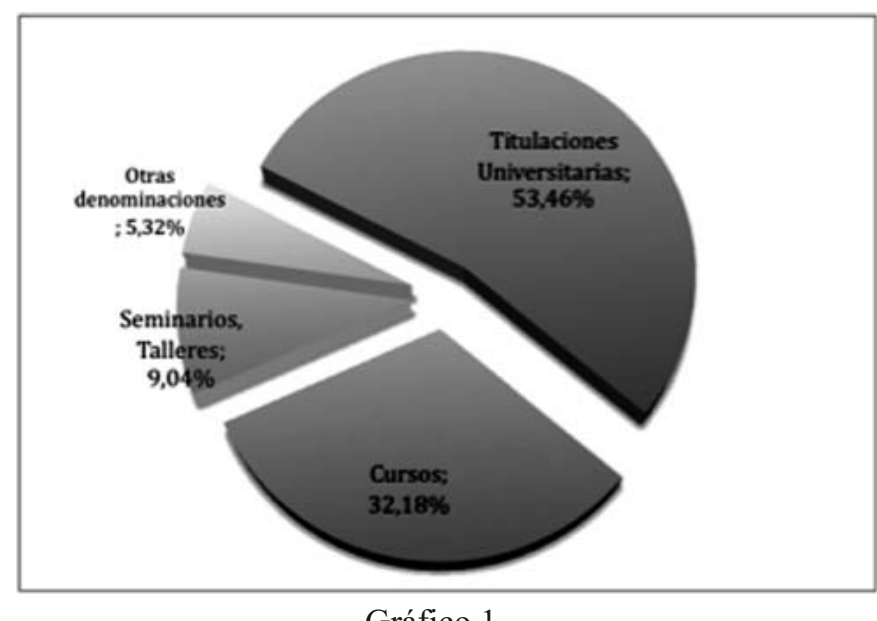

Gráfico 1

Para referirnos a las enseñanzas de carácter universitario hemos de remitirnos a la ordenación de las mismas en el marco del EEES, reguladas en nuestro país por el R.D. 1393/2007, que establece tres ciclos formativos: estudios de Grado, Máster y Doctorado, conformando los dos últimos el nivel de Posgrado. 
En Internet se ofrecen 49 titulaciones de Grado y 19 títulos descritos específicamente como Máster, si bien un amplio número de estudios (128) se enuncian genéricamente como Posgrados y cinco titulaciones universitarias no indican su nivel formativo. Se observa, pues, una preminencia de los estudios de Posgrado en Comunicación $(68,42 \%)$ frente a la oferta de Grados, que suponen solamente alrededor de la cuarta parte del total de titulaciones. Estas cifras parecen sugerir que la comunicación se está vinculando con destrezas añadidas a la formación pero que por sí sola no resulta suficiente en el ámbito laboral.

Confrontados estos datos con el catálogo de estudios universitarios de Comunicación en España, podemos advertir a priori que su presencia en la Red no es muy elevada. Sin embargo, esta observación se limita a ser una impresión general, toda vez que no se dispone de datos específicos y concretos que permitan una argumentación sólida y contrastada al respecto.

Por citar algunas de las fuentes más significativas, vemos cómo los informes Datos y cifras del Sistema Universitario que en los últimos años viene publicando periódicamente el Ministerio de Educación, ofrecen solo cifras generales, sin desagregación por áreas (número de Másteres y de programas de Doctorado en todo el territorio nacional y en todas las áreas). Los estudios de Grado se agrupan en categorías generales, de nuestro interés la de Ciencias Sociales y Jurídicas, pero igualmente resulta demasiado amplia y no ofrece datos concretos respecto al área de Comunicación (Ministerio de Educación, 2009:13-16 y 2010:18).

Tampoco el Libro Blanco Títulos de Grado en Comunicación de la ANECA (2005) sirve de referencia en este caso, puesto que describe un panorama ya superado por la integración de los estudios universitarios en el EEES. Otros trabajos afines y contemporáneos a esta investigación, como el coordinado por J. Sierra (2010) aportan información de gran interés pero en este caso demasiado específica, referida únicamente a ramas concretas de los estudios de la Comunicación (Periodismo, Relaciones Públicas, etc.).

La ausencia de investigación en este ámbito ha sido puesta también de manifiesto por Gómez-Escalonilla, Santín y Mathieu (2011: 74), cuando afirman que, a pesar de haberse desarrollado ya numerosos trabajos acerca de la enseñanza on line, "no se ha constatado ningún análisis sobre los estudios de comunicación on line". De manera que en algunos de los apartados de este trabajo nuestra aportación se ve forzosamente limitada a ofrecer una radiografía de la situación en la Red en el periodo analizado.

\subsection{Oferta formativa en Comunicación según los promotores y ejecutores de los estudios}

Un campo de interés en los estudios de la comunicación social es el estudio de los Actores y agentes sociales que intervienen en los procesos de producción y difusión del conocimiento colectivo. Por ello interesaba identificar y analizar los organismos o instituciones encargados de la promoción y la ejecución de las distintas enseñanzas.

La oferta formativa en Comunicación en Internet se vincula con tres ejecutores principales: centros de enseñanza, organizaciones empresariales y Administraciones públicas. Si bien en muchas ocasiones un único organismo ejerce conjuntamente la 
promoción y la ejecución de los estudios, ni todas las instituciones ofertan todo el abanico de estudios ni, cuando lo hacen, se dedican a todos ellos con la misma intensidad.

Los Centros de enseñanza son los principales ejecutores de los estudios universitarios $(61,18 \%$ de la oferta total), pero no los únicos, ya que también existen titulaciones promovidas por organismos empresariales $(28,89 \%)$ y, en menor medida, por las Administraciones públicas (25\%). La oferta educativa universitaria ejecutada desde la empresa y las Administraciones públicas se limita a estudios de Máster (en ambos casos en torno al $15 \%$ de su respectiva oferta formativa).

Destaca la elevada oferta de Grados de los Centros de enseñanza de titularidad pública con respecto a los privados $(25,96 \%$, frente al 9,27\%), si bien es cierto que un volumen bastante significativo de la oferta universitaria de estos últimos no especifica su nivel.

Otra diferencia significativa entre centros públicos y privados es que la oferta de Cursos, Seminarios y Talleres es mayor en los segundos, en detrimento de los estudios universitarios que son ejecutados mayoritariamente por centros públicos.

Por otra parte, la formación en formato Curso se vincula fundamentalmente al ámbito empresarial (reforzando así la tesis de que la comunicación se vincula con destrezas complementarias a la actividad principal), al tiempo que se erige como la principal apuesta formativa de la empresa $(62,50 \%$ de su oferta) en detrimento de otras opciones como Seminarios y Talleres. Una trayectoria totalmente opuesta $-\mathrm{y}$ además en proporciones similares- a la de las Administraciones públicas.

\subsection{Oferta formativa según la orientación de los estudios de Comunicación}

En sus artículos 9, 10 y 11, el Real Decreto 1393/2007 que ordena las enseñanzas universitarias oficiales perfila una orientación básica de dichos estudios: los Grados se enfocan hacia una formación general, para incorporar a los estudiantes al ejercicio profesional, mientras que las enseñanzas de Máster proporcionarán una formación avanzada orientada a la especialización académica y profesional o la actividad investigadora; finalmente, los estudios de Doctorado se encaminan hacia la formación avanzada en las técnicas de investigación y el desarrollo de la tesis doctoral.

Se ha contemplado el enfoque de los estudios desde dos vertientes: por un lado, contrastando la oferta orientada hacia el mundo académico o hacia el campo profesional; por otro, indagando sobre qué parte de esa oferta se desarrolla en una dimensión teórica y cuál desde un enfoque aplicado o práctico.

En cuanto al primero de los criterios, se advierte un alto grado de ambigüedad: en en casi la mitad de los estudios no existe un posicionamiento claro hacia ninguna de las dos opciones planteadas. Si bien, cuando sí aparece definida una orientación, el número de estudios a los cuales se les confiere una orientación profesional dobla en proporción a aquellos vehiculados hacia la enseñanza académica.

Como matiz a estos datos podría apuntarse que en muchas titulaciones principalmente de universidades privadas, la orientación no se establece a priori sino que esta viene determinada en función de las asignaturas elegidas por el alumno. Del mismo modo, debe tenerse en cuenta que la ANECA deniega la orientación académica de los Másteres de las universidades privadas si estas no demuestran una vinculación clara 
con programas de investigación académica, obligando así a un enfoque teórico y una orientación profesional.

Con respecto al segundo criterio de clasificación (enfoque teórico/práctico de los estudios), también son mayoría -aunque en proporciones menores- las acciones formativas sin una enunciación clara a este respecto. El margen de diferencia entre los estudios de orientación teórica y los aplicados -en detrimento de estos últimos-resulta aquí muy estrecho. Veamos cómo se distribuye la oferta de carácter universitario según dicha orientación.

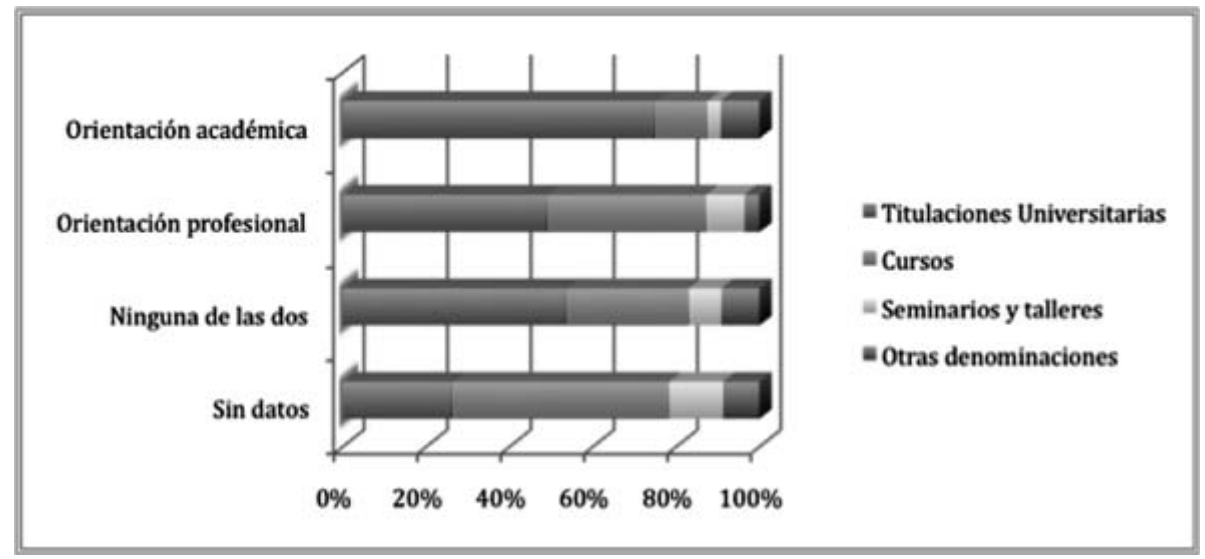

Gráfico 2

Es en los estudios universitarios donde con mayor frecuencia se menciona si se dirigen al ámbito académico o al profesional. Quizás porque es en este tipo de enseñanzas donde se lleva a cabo una formación que permite el desarrollo del alumno en este sentido, las tres cuartas partes de la oferta vinculada a una orientación académica se corresponden con titulaciones universitarias, predominantemente estudios de Grado y Máster, en idénticas proporciones. El enfoque académico prácticamente desaparece cuando nos salimos del ámbito docente universitario, según los datos de Seminarios y Talleres, Otras Denominaciones y, sobre todo, los Cursos.

Por su parte, aproximadamente una tercera parte de los estudios aluden a una orientación de tipo profesional. En este grupo se incluye un alto porcentaje de estudios universitarios (48,89\%), reflejando así el intento por parte de las Universidades de acercar al alumnado al terreno empresarial; pero también un buen número de Cursos, que emplean el recurso a las salidas laborales como reclamo para aumentar la clientela. No obstante en dos terceras partes de la oferta de Cursos no se ofrece ninguna información sobre su orientación, lo cual podría entenderse o vincularse con una cierta falta de rigor en la planificación de este tipo de ofertas docentes.

La segunda de las vertientes analizadas en cuanto a la orientación de la formación es la que considera el encauzamiento teórico o práctico de los propios estudios. En este caso la clasificación se distribuye de manera bastante uniforme entre las enseñanzas que mencionan una orientación teórica, las que ofrecen una enseñanza aplicada y aquellas otras que no se posicionan en ninguna de las dos opciones anteriores. 
Dan cuenta de este tipo de orientación fundamentalmente los estudios universitarios (al contrario de lo que ocurre en los Cursos). Y dentro de estos, resulta llamativo que los porcentajes más elevados en todas las categorías se corresponde siempre con estudios de Máster, con diferencias muy significativas respecto a las demás modalidades de educación universitaria, lo cual conduce a pensar que los programas de Máster ofrecen una información mucho más completa que los demás en lo que respecta a la orientación de los estudios.

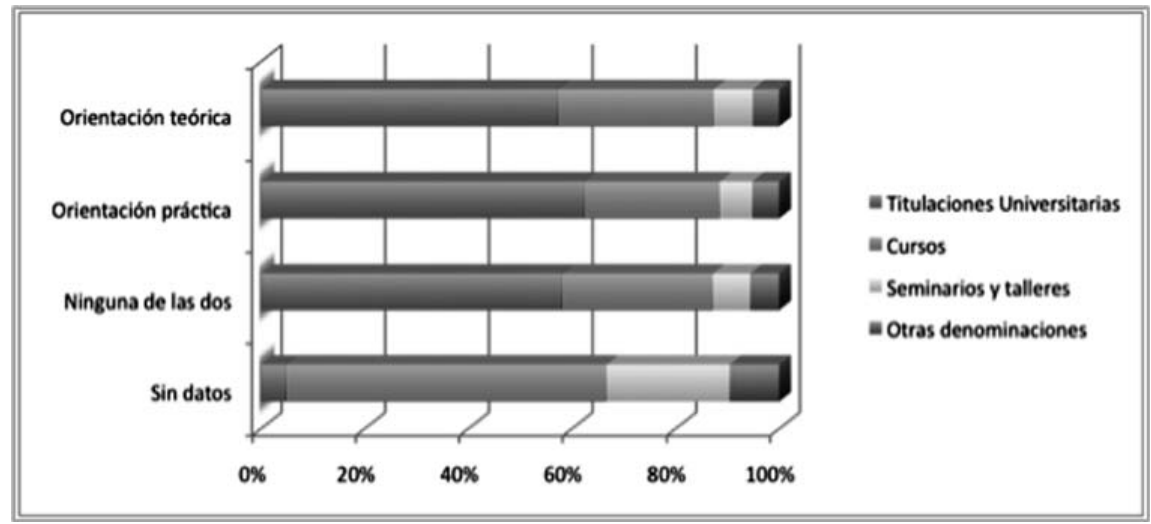

Gráfico 3

\subsection{Oferta formativa según los ámbitos de aplicación de la actividad}

En ocasiones, se aporta información que muestra una orientación de la formación hacia la práctica de alguna o de varias actividades profesionales ${ }^{2}$.

La formación en Comunicación no se aplica únicamente en entornos laborales estrictamente vinculados a los medios o sistemas de comunicación pública o social. Por ello, los ámbitos de aplicación para los cuales capacitan los estudios ofertados en la Red se han agrupado en dos bloques: áreas de trabajo relacionadas directamente con la Comunicación y Otros posibles campos de actuación profesional.

En el primer grupo se engloban los estudios diseñados para poder desempeñar una actividad profesional en campos como la Información y/o Comunicación en general, Medios de comunicación y audiencias, Marketing, Periodismo, Publicidad, Relaciones públicas, Empresas de Comunicación, Comunicación audiovisual y Otros ámbitos comunicativos ${ }^{3}$.

En el segundo bloque se agrupan las actividades profesionales en el ámbito de la Industria, Servicios, Dirección de empresas, Actividades sociales, Educación y/o Cultura, ámbito Científico, Jurídico y Otros ámbitos.

2 Sobre el análisis de los contenidos y lenguajes de los programas docentes, véase Alcoceba (2012).

${ }^{3}$ El Libro Blanco sobre los títulos de Grado en Ciencias de la Comunicación (ANECA,2005: 191-301), que ofrece los resultados de una investigación desarrollada en 2004 donde se analizaban los estudios de Comunicación que en ese momento ofrecían 40 universidades españolas, se limita a establecer los perfiles profesionales básicos en el campo de la Comunicación. 
Nuevamente, son los estudios universitarios los que en mayor medida ofrecen información relativa a su ámbito de aplicación, especialmente Grados y estudios de Máster, de manera mucho menos frecuente en estudios de Posgrado. En los demás tipos de propuestas educativas esta orientación no siempre se explicita.

A continuación se señalan algunos de los resultados más relevantes del estudio, dado que el análisis de toda la casuística encontrada excede los límites de extensión de este artículo. En cualquier caso, los gráficos 5 y 6 permitirán al lector una composición a nivel general de la situación.

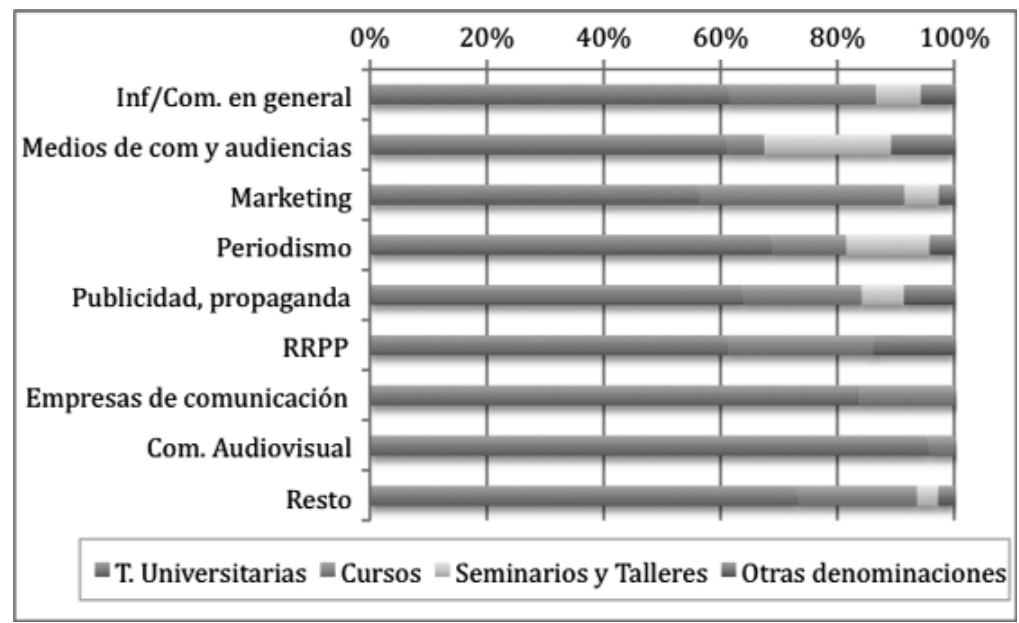

Gráfico 4

Por ejemplo, resulta llamativo que la práctica totalidad de la oferta formativa vinculada con el ejercicio profesional en Comunicación Audiovisual provenga del ámbito universitario (predominantemente Grados) y solo de manera residual aparecen Cursos con este tipo de orientación. Lo mismo ocurre con el Periodismo, mientras que los estudios en forma de Cursos aportan porcentajes muy significativos cuando se vinculan a la Comunicación en general y a Otros ámbitos.

Estos datos corroboran nuevamente la impresión general de que en la actualidad la comunicación resulta necesaria en múltiples ámbitos de la actividad profesional, desde la dirección y administración de empresas hasta la gestión de recursos humanos (en un país con un $80 \%$ de su tejido empresarial compuesto por PYMES, no es de extrañar además que los directores y gerentes de las empresas tengan que ejercer además como responsables de comunicación).

También es en los estudios universitarios donde se señala una mayor aplicación de los conocimientos en Otros ámbitos y donde se proporciona una mayor oferta de titulaciones orientadas hacia el ejercicio profesional en el área de Marketing $(56,16 \%$ de la oferta total, fundamentalmente Másteres) ${ }^{4}$.

${ }^{4}$ Un análisis más exhaustivo, derivado de la misma investigación, sobre la enseñanza aplicada hacia el Marketing puede verse en Fernández-Cid (2011). 
Por su parte, los Grados universitarios aportan los porcentajes más significativos en la oferta docente vinculada con el Periodismo, las Relaciones Públicas y la Comunicación Audiovisual, en consonancia con las tradicionales titulaciones específicas en cada uno de esos tres ámbitos.

Finalmente, la proporción más significativa de estudios vinculados a los Medios de comunicación y audiencias se encuentra en Seminarios y Talleres $(21,74 \%)$. El hecho de que estos ámbitos profesionales se estén viendo totalmente redefinidos y revolucionados por las nuevas tecnologías puede guardar relación con ello, si consideramos el uso habitual de talleres y seminarios como modalidades de reciclaje profesional para quienes han de desarrollar su actividad profesional en estos entornos tan cambiantes.

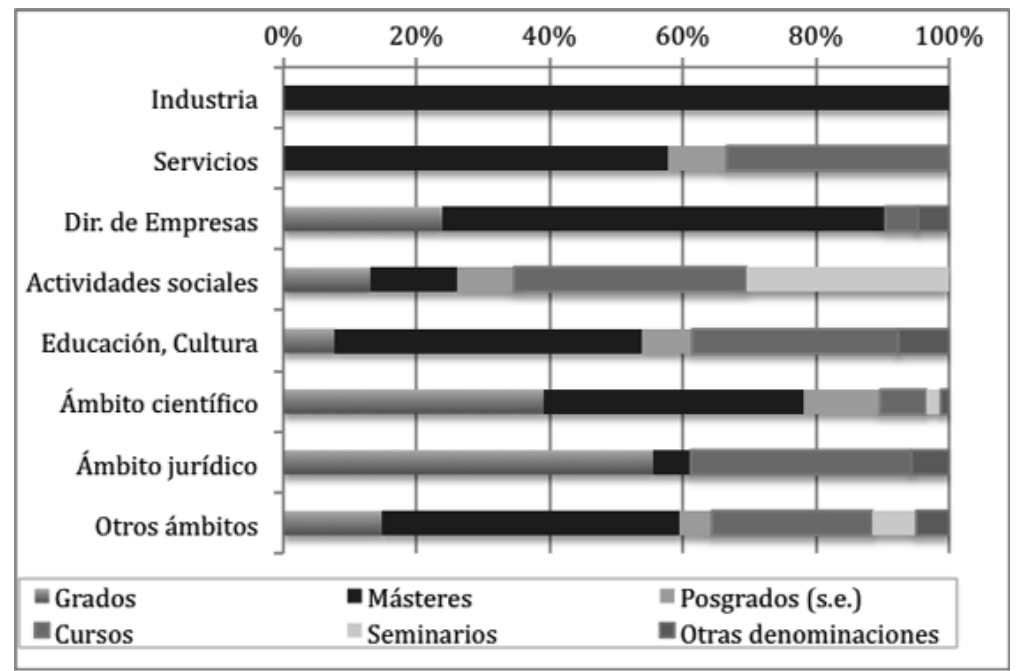

Gráfico 5

En cuanto a la oferta vinculada al ejercicio profesional en áreas de trabajo no estrictamente comunicativas, toda la relacionada con el ámbito industrial proviene de la Universidad (todos programas de Máster); y prácticamente la totalidad(89,66\%) cuando el ejercicio se destina a los entornos científico y jurídico (en estos casos también aparecen Grados).

También es el entorno universitario el que proporciona más del 90 por ciento de la oferta vinculada a la dirección de empresas (que se complementa únicamente con un pequeño volumen de Cursos) y la mayoría de la orientada hacia actividades sociales, aunque en este caso resulta igualmente significativo el número de Seminarios y Talleres.

De las acciones formativas orientadas al sector Servicios más de la mitad proviene de estudios de Máster, sin que se haya localizado ningún Grado, Seminario o Taller.

\section{Conclusiones}

La mayor parte de la oferta docente sobre Comunicación que se ofrece en Internet proviene de las Universidades, si bien es destacable el número de Cursos que se promocionan o desarrollan en la web. 
Los Centros de enseñanza públicos proporcionan un mayor número de estudios de Grado con respecto a los de titularidad privada, mientras que estos últimos ofertan un mayor volumen de Seminarios y Talleres.

Seminarios y Talleres constituyen también las acciones formativas ejecutadas mayoritariamente por las Administraciones Públicas, mientras que la empresa se declina hacia los estudios en forma de Cursos. Y la participación universitaria en ambos casos se restringe únicamente a estudios de Máster.

En cuanto a la orientación de los estudios, parecen primar las ofertas de orientación profesional frente a las académicas, aunque paradójicamente, la formación en sí parece tener un corte más teórico en detrimento de los estudios aplicados.

Analizada la oferta docente en función de los ámbitos de aplicación en los que se prevé que tengan utilidad los estudios cursados, parece que la Universidad es la que acumula la mayoría de la oferta en campos directamente relacionados con la Comunicación y sus ramas afines, lo que puede llevar a considerar que las necesidades formativas en estos ámbitos están suficientemente resueltas con las titulaciones universitarias existentes, especialmente en referencia a Periodismo, Relaciones públicas y Comunicación Audiovisual.

La oferta no universitaria en campos afines a la Comunicación se diversifica en Cursos, Seminarios, Talleres y otras denominaciones, mientras que en el caso de las disciplinas no comunicativas resulta significativa la escasez de Cursos en detrimento de la formación en forma de Seminarios y talleres.

Si nos referimos a los estudios cuyo ejercicio profesional se orienta hacia disciplinas no comunicativas (ámbito industrial, científico, jurídico, etc.), la Universidad es la única -o prácticamente la única-que ofrece formación al respecto. Es aquí donde parecen adquirir relevancia los estudios de posgrado: quizás estos resulten una opción formativa más interesante que los Grados al permitir la ampliación de competencias y el reciclaje de aquellos profesionales abocados actualmente a un cambio de sector de actividad.

\section{Referencias bibliográficas}

\section{AGENCIA NACIONAL DE EVALUACIÓN DE LA CALIDAD Y ACREDITACIÓN} (ANECA) (2005): Libro Blanco de los títulos de grado en Ciencias de la Comunicación. Madrid, ANECA

ALCOCEBA HERNANDO, José Antonio (2012): "Las propuestas tecnológicas y de contenidos de la enseñanza de la comunicación en Internet”. Diálogos de la Comunicación, Edición 84: Los Usos y las Prácticas de la Comunicación, jul. 2012, en: http://www.dialogosfelafacs.net/las-propuestas-tecnologicas-y-de-contenidosde-la-ensenanza-de-la-comunicacion-en-internet/ [fecha de consulta: 23 de agosto de 2012]

FERNÁNDEZ-CID, Matilde (2011): "La práctica de la teoría: aplicaciones profesionales de los estudios en comunicación". Investigación y Marketing, n 111, pp. 40-43.

GÓMEZ-ESCALONILLA, Gloria, SANTÍN DURÁN, Marina y MATHIEU, Gladys (2011): "La educación universitaria on-line en el Periodismo desde la visión del es- 
tudiante". Comunicar, $n^{\circ} 37$, XIX, en: http://www.revistacomunicar.com/index.php ?contenido=revista\&numero $=37$ [fecha de consulta: 23 de septiembre de 2012]

MARTÍN SERRANO, Manuel et al. (2001): Teoría de la Comunicación. I. Epistemología y análisis de la referencia. Madrid, Visor Libros

MINISTERIO DE EDUCACIÓN (2009): Datos y cifras del sistema universitario. Curso 2009/2010. Madrid, Secretaría de Publicaciones del Ministerio de Educación

MINISTERIO DE EDUCACIÓN (2011): Datos y cifras del Sistema Universitario. Curso 2011/12. Madrid, Ministerio de Educación

PEDRO, Joan (2012): “A Collection of Samples for Research in Google: Design and Application of a Sample Selection Method: Results and Problems of Research. GMJ: Mediterranean Edition, vol. 7, $\mathrm{n}^{\circ}$ 1, en: http://globalmedia.emu.edu.tr/images/stories/ALL_ARTICLES/2012/Spring/4._Joan_Pedro.pdf [fecha de consulta: 8 de septiembre de 2012].

SIERRA, Javier (Coord.) (2010): Los estudios de Ciencias de la Comunicación en el EEES. Madrid, Fragua

TRAPERO DE LA VEGA, José Ignacio (2010): “Análisis de la página web principal de las facultades de comunicación españolas", en SIERRA, Javier (Coord.): Los estudios de Ciencias de la Comunicación en el EEES. Madrid, Fragua, pp. 160-173.

\section{María CADILLA BAZ}

Universidad Complutense de Madrid

mcadilla@ccinf.ucm.es

Doctora en Ciencias de la Información y Licenciada en Documentación por la Universidad Complutense.

Profesora titular interina

Departamento de Sociología IV

Facultad de Ciencias de la Información 